\title{
Atypical Listeria innocua strains possess an intact LIPI-3
}

Evelyn M Clayton ${ }^{1 \dagger}$, Karen M Daly ${ }^{1 \dagger}$, Caitriona M Guinane ${ }^{3}$, Colin Hill ${ }^{1,2^{*}}$, Paul D Cotter ${ }^{2,3^{*}}$ and Paul R Ross ${ }^{2,3}$

\begin{abstract}
Background: Listeria monocytogenes is a food-borne pathogen which is the causative agent of listeriosis and can be divided into three evolutionary lineages I, II and III. While all strains possess the well established virulence factors associated with the Listeria pathogenicity island I (LIPI-1), lineage I strains also possess an additional pathogenicity island designated LIPI-3 which encodes listeriolysin S (LLS), a post-translationally modified cytolytic peptide. Up until now, this pathogenicity island has been identified exclusively in a subset of lineage I isolates of the pathogen Listeria monocytogenes.

Results: In total 64 L. innocua strains were screened for the presence of LIPI-3. Here we report the identification of an intact LIPI-3 in 11 isolates of $L$. innocua and the remnants of the cluster in several others. Significantly, we can reveal that placing the L. innocua lls genes under the control of a constitutive promoter results in a haemolytic phenotype, confirming that the cluster is capable of encoding a functional haemolysin.

Conclusions: Although the presence of the LIPI-3 gene cluster is confined to lineage I isolates of L. monocytogenes, a corresponding gene cluster or its remnants have been identified in many L. innocua strains.
\end{abstract}

\section{Background}

Listeria monocytogenes is a food-borne pathogen which is the causative agent of listeriosis [1-5]. It has long been known that the characteristic haemolytic phenotype of $L$. monocytogenes is attributable to the activity of listeriolysin O (LLO), encoded by the hly gene located within Listeria Pathogenicity Island I (LIPI-1) [5]. However, more recently, it has also been revealed that several strains of lineage I $L$. monocytogenes (of four evolutionary lineages, serotype $4 \mathrm{~b}$ strains within lineage I have been most commonly associated with outbreaks [6]) (also possess an additional pathogenicity island (designated LIPI-3) which encodes a second haemolysin, designated listerioly$\sin$ S [7-9]. Listeriolysin S (LLS) is not normally expressed in vitro, and hly mutants give a non-haemolytic phenotype on blood agar. LLS is one of a growing number of posttranslationally modified cytolysins (post-translationally modified haemolytic peptides) that include the Streptococcus pyogenes-associated Streptolysin S (SLS) and the

\footnotetext{
*Correspondence: c.hill@ucc.ie; paul.cotter@teagasc.ie

'Equal contributors

'Department of Microbiology, University College Cork, Cork, Ireland

${ }^{2}$ Alimentary Pharmabiotic Centre, Cork, Ireland

Full list of author information is available at the end of the article
}

Clostridium botulinum/Clostridium sporogenes-associated Clostridiolysin $\mathrm{S}$ and is a member of the broader family of thiazole/oxazole modified microcins (TOMMs) [9]. It has been established that LLS plays a role in the survival of L. monocytogenes in PMNs and also contributes to virulence in the murine model [8]. LIPI-3 consists of 8 genes arranged in the following order: IlsAGHXBYDP. LlsA is the structural peptide; LlsB, $\mathrm{Y}$ and D are enzymes proposed to perform the post-translational modifications; LlsGH is an $\mathrm{ABC}$ transporter; LlsP is a protease; while LlsX is of unknown function $[7,8]$. The associated promoter, $\mathrm{P}_{1 \mathrm{lsA}}$, which is situated upstream of $l l s A$, is not transcribed in standard laboratory media but is induced by oxidative stress. It has been suggested that expression of the LIPI-3 genes may be induced in the phagosome of macrophages [8]. When $\mathrm{P}_{\text {IlsA }}$ is replaced by a constitutive promoter $\left(\mathrm{P}_{\mathrm{HELP}}\right)$, a strongly haemolytic/cytolytic phenotype is revealed under laboratory conditions [8]. The inducible nature of LLS and its absence in many $L$. monocyctogenes strains is probably responsible for the fact that this virulence factor has gone undetected until recently.

Listeria innocua is an avirulent species within the Genus Listeria. It has been proposed that L. innocua and $L$. monocytogenes have evolved from a common ancestor
C Biomed Central 
and differ predominantly due to the loss of virulence genes by $L$. innocua $[10,11]$. This is supported by the existence of atypical L. innocua isolates which retain LIPI-1 and other virulence factors $[12,13]$. In a previous investigation we demonstrated that none of $11 \mathrm{~L}$. innocua isolates examined (one of which was initially classified as an L. grayi isolate) possessed the equivalent of the LIPI-3 $[7,8]$. In this study we extended our analysis to a larger collection of strains, which has revealed that several strains possess the remnants of a LIPI-3. In fact, 11 strains possess fully intact LIPI-3 which gives rise to a haemolytic phenotype when the genes are constitutively expressed.

\section{Methods}

\section{Strains and growth conditions}

Tables 1, 2, and 3 list the panel of Listeria strains used in this study. Strains were obtained from the Food Microbiology Microbial Collection (University College Cork) and the Special Listeria Culture Collection (SLCC). All strains were cultured at $37^{\circ} \mathrm{C}$ for $16 \mathrm{~h}$ in Brain Heart Infusion (BHI) broth or agar (Oxoid, Hampshire, UK) unless otherwise stated. Where necessary, the characterisation of strains as L. innocua was confirmed biochemically by means of the API listeria kit (BioMérieux, Lyon, France) and 16S ribosomal DNA (rDNA) with $\mathrm{CO} 1$ and $\mathrm{CO} 2$ primer pairs previously described by Simpson et al. [14]. Escherichia coli EC101 was used as an intermediate vector host. Antibiotics were incorporated as follows [8]: Erythromycin (Ery) $150 \mu \mathrm{g} / \mathrm{ml} \mathrm{E.} \mathrm{coli,} 5 \mu \mathrm{g} / \mathrm{ml}$ L. innocua. Chloroamphenicol $(\mathrm{Cm}) 10 \mu \mathrm{g} / \mathrm{ml}$ E. coli and L. innocua. Ampicillin (Amp) $100 \mu \mathrm{g} / \mathrm{ml} \mathrm{E.} \mathrm{coli.} \mathrm{5-bromo-4-chloro-3-}$ indolyl-b-D-galactopyranoside (X-Gal) was incorporated at a concentration of $40 \mu \mathrm{g} / \mathrm{ml}$.

\section{Sequence analysis}

A PCR-based strategy, employing the primer pair llsAForIlsARev, was employed to screen for the presence of the LLS structural gene, $l l s A$. These and other primers corresponding to regions both within (1113for, 1114rev, 1115 rev, 1118rev, 1120rev) and surrounding (araCrev) the LIPI-3 of L. monocytogenes F2365 were employed to amplify flanking DNA sequences which were subsequently sequenced (MWG Biotech) (Table 4). Primer Lin1080_F1, which was designed to amplify from the conserved gene, corresponding to lin1080 in strain CLIP11262, was used to determine the position of LIPI-3 in L. innocua strains relative to this locus. Overlapping sequences were assembled and a consensus sequence was determined using

Table 1 LIPI-3 positive SLCC $L$. monocytogenes strains

\begin{tabular}{|c|c|c|c|c|c|c|c|}
\hline UCC strain ID & SLCC strain ID & Lineage* & Logged date & Source & & Country of isolation & City of isolation \\
\hline 63 & SLCC4352 & I & $28 / 04 / 1975$ & Human & Spinal fluid & France & Nantes \\
\hline 74 & SLCC4563 & I & 26/11/1975 & Human & Unknown & France & Rouen \\
\hline 75 & SLCC4330 & । & $17 / 03 / 1975$ & Human & Spinal fluid & France & Nantes \\
\hline 79 & SLCC4309 & I & $14 / 02 / 1975$ & Human & Liquor & Germany & Munich \\
\hline 86 & SLCC3829 & I & $15 / 01 / 1973$ & Animal & Goat & unknown & Unknown \\
\hline 87 & SLCC3734 & I & 10/11/1972 & Food/animal & Milk & Denmark & Copenhagen \\
\hline 89 & SLCC4580 & । & $15 / 12 / 1975$ & Human & Unknown & France & Rouen \\
\hline 94 & SLCC3659 & I & $26 / 05 / 1972$ & Animal & Brain, Sheep & Germany & Frankfurt \\
\hline 101 & SLCC6254 & I & 05/06/1985 & Feed & Silage (grass) & Norway & Unknown \\
\hline 102 & SLCC6104 & । & 13/10/1984 & Environmental & Sewage & Germany & Unknown \\
\hline 105 & SLCC3733 & I & 10/11/1972 & Food/animal & Milk & Denmark & Copenhagen \\
\hline 106 & SLCC3606* & I & 06/03/1972 & Human & Unknown & Belgium & Bruxelles \\
\hline 110 & SLCC2503 & I & 1966 & Human & CFS & Germany & Stuttgart \\
\hline 113 & SLCC6088 & । & 13/10/1984 & Environmental & Sewage & Germany & Unknown \\
\hline 118 & SLCC3834 & I & 15/01/1973 & Animal & Sheep, brain & Germany & Frankfurt \\
\hline 121 & SLCC3760 & । & 24/11/1972 & Human & New born, liver & Peru & Lima \\
\hline 133 & SLCC6606 & I & 02/06/1986 & Feed & Silage & Switzerland & Unknown \\
\hline 143 & SLCC6092 & I & 13/10/1984 & Environmental & Sewage & Germany & Unknown \\
\hline 148 & SLCC3732 & । & 10/11/1972 & Food/animal & Milk & Denmark & Copenhagen \\
\hline 154 & SLCC3106 & I & 09/02/1970 & Human & Liquor & Germany & Idar-Oberstein \\
\hline 156 & SLCC4157 & I & 09/05/1974 & Animal & Cow, Brain & Germany & Freiburg \\
\hline
\end{tabular}

*Lineages revealed by allele specific oligonucleotide (ASO)-PCR [15]. 
Table 2 IIsA negative L. monocytogenes strains

\begin{tabular}{|c|c|c|c|c|c|c|c|}
\hline UCC strain ID & SLCC ID & Lineage* & Logged date & Source & & Country of isolation & City of isolation \\
\hline 64 & SLCC3996 & I & $31 / 08 / 1973$ & Human & Spinal fluid & France & Nantes \\
\hline 65 & SLCC4410 & $\|$ & 15/07/1975 & Human & Blood & France & Nantes \\
\hline 66 & SLCC4068 & $\|$ & 08/01/1973 & Animal & Red deer, faeces & Germany & Freiburg \\
\hline 67 & SLCC6303 & $\|$ & 05/06/1985 & Feed & Silage (grass) & Norway & Unknown \\
\hline 68 & SLCC6374 & $\|$ & 05/06/1985 & Feed & Silage (grass) & Norway & Unknown \\
\hline 69 & SLCC6342 & $\|$ & 05/06/1985 & Feed & Silage & Norway & Unknown \\
\hline 70 & SLCC4274 & I & 26/11/1974 & Human & Unknown & Germany & Freiburg \\
\hline 71 & SLCC4280 & $\|$ & $16 / 12 / 1974$ & Unknown & Unknown & Slovak Republic & Bratislava \\
\hline 73 & SLCC4063 & $\|$ & 08/01/1974 & Animal & Cattle, faeces & Germany & Freiburg \\
\hline 76 & SLCC4349 & $\|$ & 28/04/1975 & Human & Blood & France & Nantes \\
\hline 77 & SLCC4290 & $\|$ & 16/12/1974 & Unknown & Unknown & Slovak Republic & Bratislava \\
\hline 78 & SLCC4100 & $\|$ & 05/03/1974 & Animal & Sheep, brain & Germany & Stuttgart \\
\hline 80 & SLCC4481 & $\|$ & 27/10/1975 & Unknown & Unknown & Spain & Madrid \\
\hline 81 & SLCC4077 & $\|$ & $15 / 02 / 1974$ & Human & Blood & France & Nantes \\
\hline 82 & SLCC3852 & $\|$ & 09/04/1973 & Animal & Lamb, brain & Germany & Stuttgart \\
\hline 83 & SLCC4235 & $\|$ & 16/09/1974 & Animal & Hare, caecum & Denmark & Copenhagen \\
\hline 84 & SLCC4209 & $\|$ & $12 / 08 / 1974$ & Human & Intestine & Germany & Heidelberg \\
\hline 85 & SLCC4230 & $\|$ & 16/09/1974 & Animal & Hare, caecum & Denmark & Copenhagen \\
\hline 88 & SLCC4592 & $\|$ & 15/12/1975 & Human & Unknown & France & Rouen \\
\hline 93 & SLCC3738 & $\|$ & 10/11/1972 & Animal & Horse & Denmark & Copenhagen \\
\hline 95 & SLCC4455 & $\|$ & 10/09/1975 & Unknown & Unknown & Hungary & Szolnok \\
\hline 96 & SLCC4439 & $\|$ & 10/09/1975 & Unknown & Unknown & Hungary & Szolnok \\
\hline 97 & SLCC4315 & I & $14 / 02 / 1975$ & Human & Liquor & Australia & North Adelaide \\
\hline 98 & SLCC4234 & $\|$ & 16/09/1974 & Animal & Hare, caecum & Denmark & Copenhagen \\
\hline 99 & SLCC6108 & । & 13/10/1984 & Environmental & Sewage & Germany & Unknown \\
\hline 100 & SLCC643 & $\|$ & 01/01/1958 & Human & csf & USA & Georgia \\
\hline 103 & SLCC6340 & $\|$ & 05/06/1985 & Feed & Silage & Norway & Unknown \\
\hline 104 & SLCC293 & III & 01/01/1955 & Unknown & Unknown & USA & Maryland \\
\hline 107 & SLCC3631 & $\|$ & $12 / 04 / 1972$ & Animal & Sheep, brain & Germany & Frankfurt \\
\hline 108 & SLCC2671 & III & 01/01/1967 & Unknown & Unknown & USA & California \\
\hline 109 & SLCC2634 & III & 1934 & Animal & Ruminant & USA & Unknown \\
\hline 111 & SLCC6255 & $\|$ & 05/06/1985 & Feed & Silage (grass) & Norway & Unknown \\
\hline 112 & SLCC6202 & $\|$ & 05/06/1985 & Feed & Silage (grass) & Norway & Unknown \\
\hline 114 & SLCC6605 & $\|$ & 02/06/1986 & Feed & Silage (maize) & Switzerland & Unknown \\
\hline 115 & SLCC4138 & $\|$ & 23/04/1974 & Animal & Lymph node & Togo & Lome \\
\hline 116 & SLCC4617 & $\|$ & 28/12/1975 & Unknown & Unknown & Switzerland & Basel \\
\hline 117 & SLCC4618 & $\|$ & 28/12/1975 & Unknown & Unknown & Switzerland & Basel \\
\hline 119 & SLCC4101 & $\|$ & 05/03/1974 & Animal & Sheep, brain & Germany & Stuttgart \\
\hline 120 & SLCC4070 & $\|$ & 08/01/1974 & Animal & Cattle, faeces & Germany & Freiburg \\
\hline 123 & SLCC3939 & $\|$ & 09/07/1973 & Human & Blood & Belgium & Bruxelles \\
\hline 125 & SLCC3847 & $\|$ & 09/04/1973 & Animal & Fox, brain & Slovenia & Ljubljana \\
\hline 125 & SLCC3864 & $\|$ & 09/04/1973 & Animal & Calf, organs & Germany & Freiburg \\
\hline 126 & SLCC4079 & $\|$ & $15 / 02 / 1974$ & Human & Meconium & France & Nantes \\
\hline 127 & SLCC4294 & $\|$ & 16/12/1974 & Unknown & Unknown & Slovak Republic & Bratislava \\
\hline
\end{tabular}


Table 2 IIsA negative L. monocytogenes strains (Continued)

\begin{tabular}{|c|c|c|c|c|c|c|c|}
\hline 128 & SLCC4442 & $\|$ & 10/09/1975 & Unknown & Unknown & Hungary & Szolnok \\
\hline 129 & SLCC4444 & $\|$ & 10/09/1975 & Unknown & Unknown & Hungary & Szolnok \\
\hline 130 & SLCC3278 & I & 03/09/1970 & Animal & Duck, liver & Denmark & Copenhagen \\
\hline 131 & SLCC3270 & $\|$ & 03/09/1970 & Animal & Hare, pus & Denmark & Copenhagen \\
\hline 132 & SLCC3258 & $\|$ & 02/09/1970 & Unknown & Unknown & Belgium & Bruxelles \\
\hline 135 & SLCC5203 & $\|$ & 17/11/1977 & Feed & Silage & Netherlands & Unknown \\
\hline 136 & SLCC3683 & $\|$ & 22/06/1972 & Environmental & Fir needle & Germany & Unknown \\
\hline 137 & SLCC6611 & $\|$ & 02/06/1986 & Environmental & Soil & Switzerland & Unknown \\
\hline 138 & SLCC4153 & 1 & 09/05/1974 & Animal & Faeces & Germany & Freiburg \\
\hline 139 & SLCC3269 & $\|$ & 03/09/1970 & Animal & Hare, spleen & Denmark & Copenhagen \\
\hline 141 & SLCC3214 & $\|$ & 18/06/1970 & Human & Spinal fluid & France & Lyon \\
\hline 144 & SLCC6343 & $\|$ & 05/06/1985 & Feed & Silage & Unknown & Unknown \\
\hline 146 & SLCC3629 & । & $04 / 04 / 1972$ & Human & New born; intestine, liver & Peru & Lima \\
\hline 147 & SLCC3569 & $\|$ & 08/02/1972 & Animal & Hen & France & Alfort \\
\hline 149 & SLCC3458 & । & 08/07/1971 & Human & Unknown & France & Rouen \\
\hline 150 & SLCC3457 & $\|$ & 08/07/1971 & Human & Unknown & France & Rouen \\
\hline 152 & SLCC3366 & । & $11 / 03 / 1971$ & Animal & Pig, brain & Germany & Freiburg \\
\hline 153 & SLCC3277 & $\|$ & 03/09/1970 & Animal & Bird, liver & Denmark & Copenhagen \\
\hline
\end{tabular}

*Lineages revealed by allele specific oligonucleotide (ASO)-PCR [15].

the Seqmanager programme (Lasergene 6) and deposited in Genbank (accession numbers KJ394487, KJ394488, KJ394489 and KJ394490). Putative open reading frames (ORFs) were identified and pair-wise alignment of protein sequences was carried out using Needlemann-Wunsch global alignment algorithms accessed via the European Bioinformatics Institute (EBI) web server. Shading of multiple-aligned sequences was carried out using the Boxshade programme (version 3.2) accessed via the European Molecular Biology web server (EMBnet).

\section{Constitutive expression of the LIPI-3 cluster of $L$. innocua strain FH2051}

The L. innocua FH2051 lls genes were placed under the control of the strong constitutive synthetic promoter $\mathrm{P}_{\text {HELP }}$ using the pORI-based repA-negative plasmid system as previously described by Cotter et al., with some modification [8]. Briefly, $P_{\text {HELP }}$ DNA was amplified with the primer pair PhelpFsoe(LI)/PhelpRsoe from the plasmid pPL2luxPHelp [16] and fused between two DNA fragments amplified from the regions flanking $\mathrm{P}_{l l s A}$ by splicing by overlap extension (SOE) PCR [17]. The upstream region was amplified with the primer pair PllsAchgA(LI) and PllsAchgB(LI) and the downstream region was amplified with primers PllsAchgC and PllsAchgD. All PCRs were performed using Vent DNA polymerase (NEB, New England Biolabs, MA, USA). The SOE PCR product was cloned into the multiple cloning site (MCS) of pORI280 following PstI and EcoRI (NEB) digestion and ligation with the Ligafast rapid DNA ligation system (Promega, Madison, USA). The sequence of the cloned product was verified with MCS primers pORI280For/Rev by MWG Biotech, Germany [18]. Pellet-paint (Novagen) precipitated plasmid was subsequently transformed into the intermediate repA-positive host E. coli EC101. The plasmid was co-transformed into L. innocua FH2051 with the highly temperature-sensitive plasmid pVE6007 which supplies RepA in trans. Transformed cells appeared as blue colonies following plating on BHI-Ery-Xgal at $30^{\circ} \mathrm{C}$. The integration of pORI280 by single crossover homologous recombination was stimulated by picking a single blue colony from the transformation plate and incubating it on BHI-Ery-Xgal at $30^{\circ} \mathrm{C}$ for $24 \mathrm{~h}$ and subcultured twice on $\mathrm{BHI}-$ Ery-Xgal at $42^{\circ} \mathrm{C}$. A second crossover event, resulting in the introduction of $\mathrm{P}_{\text {HELP }}$ in place of $\mathrm{P}_{\text {llsA }}$ and the eventual loss of the pORI280 vector, was screened for following multiple subcultures in the absence of antibiotic selection. The introduction of $\mathrm{P}_{\text {HELP }}$ upstream of $l l s A$ in Ery resistant $\mathrm{Cm}$ sensitive colonies was confirmed by PCR. A haemolytic phenotype was determined by spotting $10 \mu \mathrm{L}$ of an overnight culture of this strain onto Columbia blood agar (Oxoid) containing 5\% defibrinated horse blood (TCS Biosciences, Buckingham, UK) and $1 \mathrm{mU} / \mathrm{ml}$ sphingomyelinase (Sigma) and examining after $24 \mathrm{~h}$.

\section{Pulsed- field gel electrophoresis}

Pulsed-field gel electrophoresis was carried out following the CDC standardized PulseNet protocol for 
Table 3 Listeria innocua strains used in this study

\begin{tabular}{|c|c|c|c|c|c|c|c|c|c|}
\hline UCC strain ID & SLCC strain ID & Serotype & Logged date & Source & & $\begin{array}{l}\text { Country of } \\
\text { isolation }\end{array}$ & $\begin{array}{l}\text { City of } \\
\text { isolation }\end{array}$ & IIsA PCR & $\begin{array}{l}\text { LIPI-3 } \\
\text { PCR }\end{array}$ \\
\hline 1 & SLCC7157* & $6 a$ & 08/12/1986 & Animal & Roe & Switzerland & Bern & $\checkmark$ & $x$ \\
\hline 2 & SLCC7199 & $6 b$ & 18/12/1986 & Food & Cheese & Germany & Munich & $\checkmark$ & $\checkmark$ \\
\hline 3 & SLCC6483 & $6 b$ & 05/03/1986 & Food & Cheese & Switzerland & St.Gallen & $x$ & $x$ \\
\hline 4 & SLCC6109 & $6 a$ & 13/10/1984 & Sewage & Sewage & Germany & Braunschweig & $x$ & $x$ \\
\hline 5 & SLCC6814 & $4 c$ & 07/05/1986 & Human & Liquor (meningitis) & UK & London & $\checkmark$ & $\checkmark$ \\
\hline 6 & SLCC6270 & $6 b$ & 05/06/1985 & Animal & Goat & Norway & Minde & $\checkmark$ & $x$ \\
\hline 7 & SLCC6276 & $6 b$ & 05/06/1985 & Animal & Sheep & Norway & Minde & $\checkmark$ & $\checkmark$ \\
\hline 8 & SLCC6362 & $6 b$ & 05/06/1985 & Animal & Sheep & Norway & Minde & $\checkmark$ & $x$ \\
\hline 9 & SLCC6370* & $6 b$ & 05/06/1985 & Animal & Sheep & Norway & Minde & $\checkmark$ & $x$ \\
\hline 10 & SLCC6382 & $6 b$ & 05/06/1985 & Animal & Sheep & Norway & Minde & $\checkmark$ & $x$ \\
\hline 11 & SLCC6285* & $6 b$ & 05/06/1985 & Feed & Silage (grass) & Norway & Minde & $\checkmark$ & $x$ \\
\hline 12 & SLCC6373 & $6 b$ & 05/06/1985 & Feed & Silage (grass) & Norway & Minde & $\checkmark$ & $x$ \\
\hline 13 & SLCC6098 & $6 a$ & 13/10/1984 & Sewage & Sewage & Germany & Braunschweig & $x$ & $x$ \\
\hline 14 & SLCC6007 & $6 a$ & 10/08/1984 & & & Brasil & Rio de Janeiro & $x$ & $x$ \\
\hline 15 & SLCC6099 & $6 a$ & 13/10/1984 & Sewage & Sewage & Germany & Braunschweig & $x$ & $x$ \\
\hline 16 & SLCC6364 & $6 b$ & 05/06/1985 & Animal & Sheep & Norway & Minde & $\checkmark$ & $x$ \\
\hline 17 & SLCC6317* & $6 b$ & 05/06/1985 & Animal & Sheep & Norway & Minde & $\checkmark$ & $x$ \\
\hline 18 & SLCC7030 & $6 a$ & 14/11/1986 & Food & Cheese & Germany & Munich & $\checkmark$ & $x$ \\
\hline 19 & SLCC6297* & $6 b$ & 05/06/1985 & Feed & Silage (grass) & Norway & Minde & $\checkmark$ & $x$ \\
\hline 20 & SLCC6356 & $6 b$ & 05/06/1985 & Food/animal & Milk & Norway & Minde & $\checkmark$ & $x$ \\
\hline 21 & SLCC6235 & $6 b$ & 05/06/1985 & Silage (grass) & Silage (grass) & Norway & Minde & $\checkmark$ & $x$ \\
\hline 22 & SLCC6298 & $6 b$ & 05/06/1985 & Feed & Silage (grass) & Norway & Minde & $\checkmark$ & $\checkmark$ \\
\hline 23 & SLCC6203 & $6 b$ & 05/06/1985 & Silage (grass) & Silage (grass) & Norway & Minde & $\checkmark$ & $\checkmark$ \\
\hline 24 & SLCC7116 & $6 a$ & 17/11/1986 & Food & Cheese & Austria & Innsbruck & $\checkmark$ & $x$ \\
\hline 25 & SLCC6353 & $6 b$ & 05/06/1985 & Food/animal & Milk & Norway & Minde & $\checkmark$ & $x$ \\
\hline 26 & SLCC6409 & $6 b$ & 05/06/1985 & Feed & Silage (grass) & Norway & Minde & $\checkmark$ & $x$ \\
\hline 28 & SLCC6541 & $6 a$ & 23/04/1986 & Food & Cheese & Germany & Munich & $\checkmark$ & $x$ \\
\hline 29 & SLCC6927 & $6 b$ & 22/09/1986 & & & Austria & Vienna & $\checkmark$ & $x$ \\
\hline 31 & SLCC6228 & $6 b$ & 05/06/1985 & Silage (grass) & Silage (grass) & Norway & Minde & $\checkmark$ & $x$ \\
\hline 30 & SLCC6749 & $6 b$ & $31 / 07 / 1986$ & Food & Cheese & Germany & Munich & $\checkmark$ & $\checkmark$ \\
\hline 32 & SLCC6322 & $6 a$ & 05/06/1985 & Feed & Silage (grass) & Norway & Minde & $\checkmark$ & $x$ \\
\hline 33 & SLCC5916 & $6 a$ & 16/03/1984 & & & Switzerland & Lausanne & $\checkmark$ & $x$ \\
\hline 34 & SLCC5326 & $6 a$ & 09/03/1979 & & & USA & Richmond, Virginia & $\checkmark$ & $x$ \\
\hline 35 & SLCC6283 & $6 b$ & 05/06/1985 & Feed & Silage (grass) & Norway & Minde & $\checkmark$ & $x$ \\
\hline 36 & SLCC6246 & $6 b$ & 05/06/1985 & Feed & Silage (grass) & Norway & Minde & $\checkmark$ & $x$ \\
\hline 37 & SLCC3533 & $4 \mathrm{~b}$ & $06 / 12 / 2010$ & Environment & Leaves & Germany & Freiburg & $x$ & $x$ \\
\hline 38 & SLCC6466 & $6 b$ & 30/01/1986 & Food & Cheese & Switzerland & St.Gallen & $\checkmark$ & $\checkmark$ \\
\hline 39 & SLCC6359 & $6 b$ & 05/06/1985 & Animal & Goat & Norway & Minde & $\checkmark$ & $x$ \\
\hline 40 & SLCC6286 & $6 b$ & 05/06/1985 & Feed & Silage (grass) & Norway & Minde & $\checkmark$ & $x$ \\
\hline 41 & SLCC6294 & $6 b$ & 05/06/1985 & Animal & Sheep & Norway & Minde & $\checkmark$ & $\checkmark$ \\
\hline 42 & SLCC6371 & $6 b$ & 05/06/1985 & Animal & Sheep & Norway & Minde & $\checkmark$ & $x$ \\
\hline 43 & SLCC6119 & $6 a$ & 10/12/1984 & Human & & Germany & Goettingen & $x$ & $x$ \\
\hline 44 & SLCC3947 & $4 f$ & 27/07/1973 & Human & & Germany & Cologne & $x$ & $x$ \\
\hline
\end{tabular}


Table 3 Listeria innocua strains used in this study (Continued)

\begin{tabular}{|c|c|c|c|c|c|c|c|c|c|}
\hline 45 & SLCC6519 & $6 a$ & 23/03/1986 & Food & Cheese & Germany & Munich & $x$ & $x$ \\
\hline 46 & SLCC6408* & $6 b$ & 05/06/1985 & Feed & Silage (grass) & Norway & Minde & $\checkmark$ & $x$ \\
\hline 47 & SLCC6296 & $6 b$ & 05/06/1985 & Feed & Silage (grass) & Norway & Minde & $\checkmark$ & $\checkmark$ \\
\hline 48 & SLCC5328 & $6 b$ & 09/03/1979 & & & USA & Richmond, Virginia & $x$ & $x$ \\
\hline 49 & SLCC6279 & $6 b$ & 05/06/1985 & Animal & Sheep & Norway & Minde & $\checkmark$ & $\checkmark$ \\
\hline 50 & SLCC6318 & $6 b$ & 05/06/1985 & Animal & Sheep & Norway & Minde & $x$ & $x$ \\
\hline 51 & SLCC6542 & $6 a$ & 23/04/1986 & Food & Cheese & Germany & Munich & $\checkmark$ & $x$ \\
\hline 52 & SLCC6272 & $6 b$ & 05/06/1985 & Animal & Goat & Norway & Minde & $\checkmark$ & $x$ \\
\hline 53 & SLCC3835* & $6 b$ & 08/02/1973 & Human & & Germany & Cologne & $\checkmark$ & $x$ \\
\hline 54 & SLCC5998 & $6 b$ & 16/07/1984 & Animal & Cattle & Belgium & Bruxelles & $x$ & $x$ \\
\hline 55 & SLCC6670 & $6 a$ & 02/06/1986 & Food & Milk & Switzerland & Bern & $x$ & $x$ \\
\hline 56 & SLCC6667 & $6 a$ & 02/06/1986 & Food & Milk & Switzerland & Bern & $x$ & $x$ \\
\hline 57 & SLCC5753* & $6 b$ & 16/11/1982 & & & Slovak Republic & Bratislava & $\checkmark$ & $x$ \\
\hline 58 & SLCC7113 & $6 b$ & 17/11/1986 & Food & Cheese & Austria & Vienna & $x$ & $x$ \\
\hline 59 & SLCC6103 & $6 b$ & 13/10/1984 & Sewage & Sewage & Germany & Braunschweig & $x$ & $x$ \\
\hline 60 & SLCC6543 & $6 a$ & 23/04/1986 & Food & Cheese & Germany & Munich & $\checkmark$ & $x$ \\
\hline 61 & SLCC6977* & $4 c$ & 13/10/1986 & Food & Cheese & Germany & Munich & $\checkmark$ & $\checkmark$ \\
\hline 62 & SLCC6921 & $6 a$ & 22/09/1986 & Food & Milk & Switzerland & Bern & $x$ & $x$ \\
\hline $\mathrm{FH} 2034$ & N/A & Unknown & 2000 & Food & Raw mince & Ireland & Cork & $\checkmark$ & $x$ \\
\hline FH1836 & $\mathrm{N} / \mathrm{A}$ & Unknown & 2000 & Food & Spinach cannelloni & Ireland & Cork & $\checkmark$ & $x$ \\
\hline FH2051 & N/A & Unknown & 2000 & Food & Chicken nuggets & Ireland & Cork & $\checkmark$ & $x$ \\
\hline
\end{tabular}

*Possess IIsA but not other LIPI-3 associated genes.

L. monocytogenes with AscI and ApaI as the restriction endonucleases. The PFGE patterns were analyzed using BioNumerics software [19].

\section{Results and discussion}

\section{Screening $L$. monocytogenes and $L$. innocua for homologues of IIsA}

To date LIPI-3 has been identified in $\sim 60 \%$ (27 of 46 ) of lineage I L. monocytogenes but was absent from all lineage II $(n=23)$ and lineage III $(n=5)$ isolates tested [8]. As a consequence of gaining access to the Seeliger collection of Listeria isolates [20], we were provided with the opportunity to screen for the presence of LIPI-3 among an additional $83 \mathrm{~L}$. monocytogenes isolates including 30 lineage I, 50 lineage II and 3 lineage III strains. The lls $A$ gene was not identified in any lineage II or lineage III strain, consistent with our previous observations (Table 1). However, the $l l s A$ gene was identified in $70 \%$ of lineage I L. monocytogenes screened (21 of 30) and, on the basis of PCR amplification, in all cases the full complement of LIPI-3 genes was present. All such isolates originated from human, animal (including milk and feed) and sewage sources. When collated with data from previous studies, it is apparent that $63 \%$ (48 of 76 ) of lineage I isolates are LIPI-3 positive and may be capable of LLS production. All LIPI-3 positive isolates belonged to Lineage $\mathrm{I}$ as verified by an allele specific oligonucleotide PCR multiplex (actA1-f, actA1-r, plcB2-f, plcB2-r, actA3-f, plcB3-r) based on the prfA virulence gene cluster [15], thus verifying previous observations with respect to the distribution of LIPI-3 among different evolutionary lineages of L. monocytogenes $[7,8]$.

Access to the Seeliger collection and other strains also facilitated a further investigation of the LIPI-3 status of L. innocua. As stated, a previous analysis of 11 strains of L. innocua indicated that all lacked genes associated with LIPI-3 $[7,8]$. However, screening a larger collection of 64 L. innocua strains using $l l s A$ specific primers revealed that 45 strains (70.3\%) were $l l s A$-positive (Table 3). Further PCR-based analysis of these isolates, employing a variety of primers designed to amplify across and within the LIPI-3 (llsAFor, llsARev, 1113for, 1114rev, 1115rev, 1118rev, 1120 rev, araCrev) revealed that 11 of these strains possess a cluster which is comparable in size, gene content and gene organisation to that of the LIPI-3 cluster found in a subset of lineage I L. monocytogenes strains. These 11 isolates originated from a number of European countries between 1984 and 2000, and were isolated from varied sources including processed chicken [1], cheese [7], sheep [7], silage [7] and human [1] (Table 3). Further analysis revealed that $25 \mathrm{~L}$. innocua isolates possess a truncated LIPI-3 with no PCR product generated for $l l s B Y D P$. Sequencing the region confirmed that these genes are absent 
Table 4 Primers used in this study

\begin{tabular}{|c|c|}
\hline Primer name & Sequence $\left(5^{\prime} \text { to } 3^{\prime}\right)^{*}$ \\
\hline PllsAchgA(LI) & GGCTGCAGAATCCGCGTTCTTG \\
\hline PllsAchgB(LI) & GAGGTTTTAGGGCTTTGCTC \\
\hline PhelpFsoe(LI) & GAGCAAAGCCCTAAAACCTCGAGATCTGCTGG \\
\hline PhelpRsoe & GATGATTGTGATTTAATATTCATGGGTTTCACTCTC \\
\hline PllsAchgC & ATGAATATTAAATCACAATCATC \\
\hline PllsAchgD & TGGAATTCCCAGCTCCATTGTCTC \\
\hline pORI280For & СTCGTTCATTATAACCCTC \\
\hline pORI280Rev & CGCTTCCTTTCCCCCCAT \\
\hline Lin1080_F1 & CGGTACGGATTGTGAATGAAGTGG \\
\hline IlsAFor & CGATTTCACAATGTGATAGGATG \\
\hline IIsARev & GCACATGCACCTCATAAC \\
\hline 1113 for & GTTATGAGGTGCATGTGC \\
\hline 1114rev & GTCTGGGATATGTAGTCC \\
\hline $1115 \mathrm{rev}$ & CACTAGCATGATGTTTATAGGGG \\
\hline 1118rev & CATGACAAGCAGTGCCTGTTGATACAGC \\
\hline 1120rev & CGTTCCCCCTCCTTTTAAGAGCAG \\
\hline araCrev & СTCTCCTITTCATTAGCCTGC \\
\hline actA1-f & AATAACAACAGTGAACAAAGC \\
\hline actA1-r & TATCACGTACCCATTTACC \\
\hline plcB2-f & TTGTGATGAATACTTACAAAC \\
\hline plcB2-r & TTGCTACCATGTCTTCC \\
\hline actA3-f & CGGCGAACCATACAACAT \\
\hline plcB3-r & TGTGGTAATTTGCTGTCG \\
\hline
\end{tabular}

*Restriction site in bold and SOE overhang italicised. in at least two isolates (SLCC6270 and SLCC6382). With the exception of $l l s P$, these genes have previously been found to be essential for LLS production in L. monocytogenes [7]. Of the remaining 28 strains, 9 were found to contain $l l_{s} A$ but attempts to amplify across or within other LIPI-3 associated genes were unsuccessful and another 19 isolates lacked all LIPI-3 genes.

Two L. innocua isolates, SLCC6382 and SLCC6270, containing a truncated LIPI-3, were selected for further analysis. Both SLCC6382 and SLCC6270 shared 98\% homology with respect to the structural peptide LlsA. The putative LlsG, LlsH and LlsX proteins from both strains shared 96\%, 99\% and 95\% identity with their L. monocytogenes counterpart. $l l s B, l l s Y, l l s D$ and $l l s P$ are absent from both isolates, while the AraC-like regulatory protein determinant was present with $98 \%$ identity to the L. monocytogenes cluster. As in L. monocytogenes, the L. innocua cluster is located downstream of a putative glutamine hydrolyzing GMP synthase protein (GuaA). However, the island in SLCC6382 and SLCC6270 commences 600 bases immediately downstream of guaA and thus is not flanked by glyoxylase encoding genes, thereby contrasting with LIPI-3 in L. monocytogenes.

Three strains (SLCC6466, SLCC6294, FH2051) possessing an entire LIPI-3 cluster were also selected for a more extensive investigation. Eight complete ORFs were identified, each corresponding to their homologue in the L. monocytogenes LIPI-3 cluster (llsAGHXBYDP). Sequence alignments confirmed considerable homology at the protein level (Figure 1). The structural peptide LlsA shared

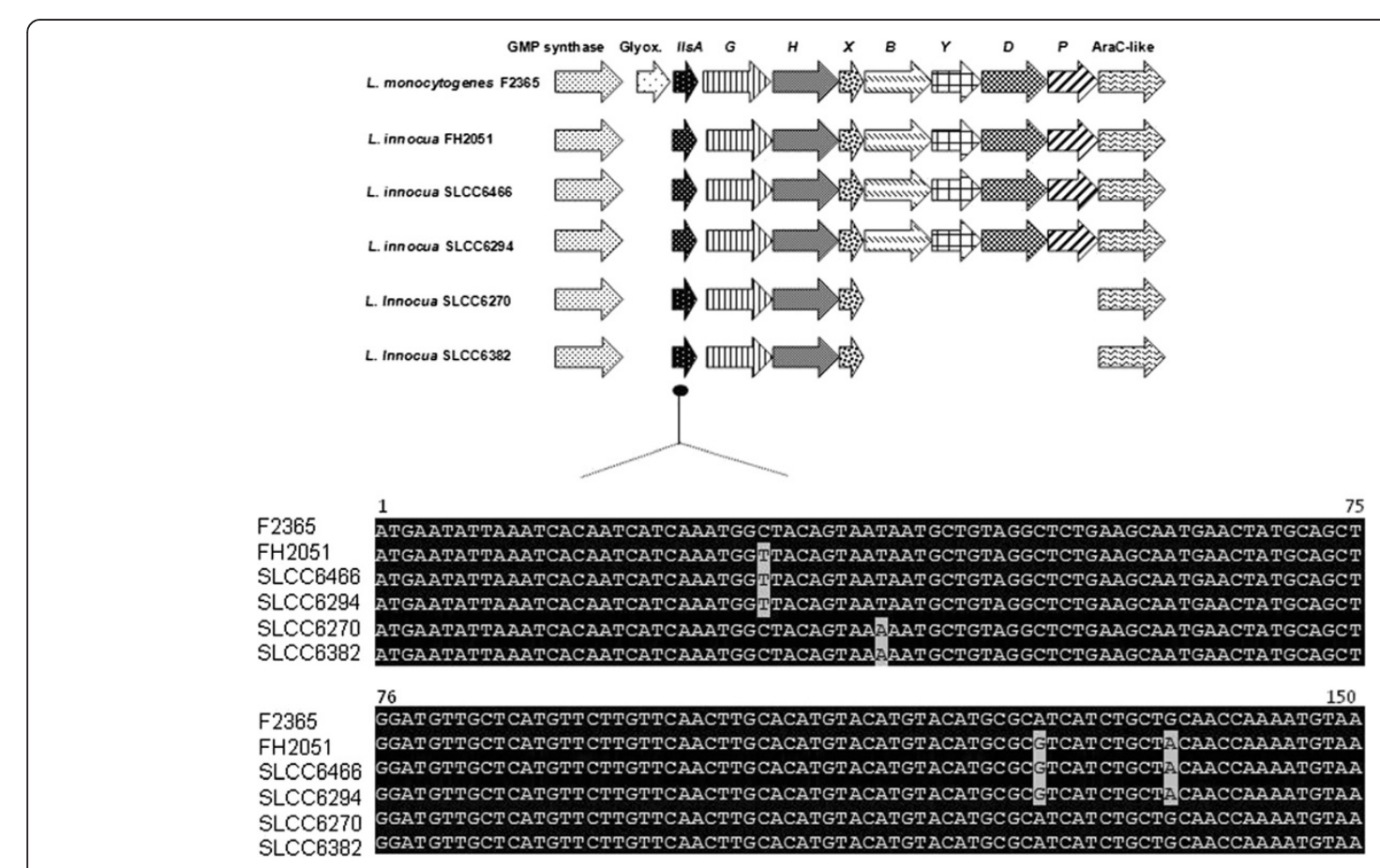

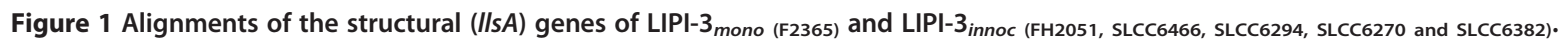




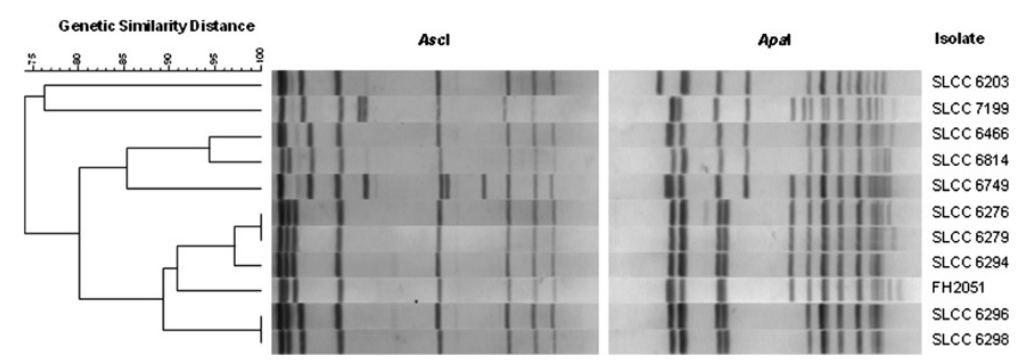

Figure 2 Dendrograms derived from PFGE profiles of Ascl and Apafl macrorestriction displaying restriction pattern similarity among the $11 \mathrm{~L}$. innocua LIPI-3 ${ }^{+}$isolates.

98\% homology in the case of the three strains mentioned above to the $L$. monocytogenes equivalent. These L. innocua clusters also encode homologs of the putative two component $\mathrm{ABC}$ transport system LlsG and LlsH, with LlsG sharing 95.3\% (FH2051) and 95\% (SLCC6466, SLCC6294) identity, and 98.8\% (FH2051) and 99\% (SLCC6466, SLC C6294) with respect to LlsH. The putative LlsX homolog, which is of unknown function, is $97 \%$ identical to its L. monocytogenes counterpart for all three isolates. This gene is believed to be specific to LIPI-3 since no homologue exists among other sag-like gene clusters [7]. A corresponding cluster of putative Lls homologs, all of which are predicted to encode biosynthetic enzymes, were also identified [8]; LlsB ( $99 \%$ in the case of all three strains), LlsY (95.4\% FH2051, 95\% SLCC6466 and SLCC6294) and LlsD (98.4\% FH2051, 98\% SLCC6466 and SLCC6294). Finally, the L. innocua cluster also carries putative LlsP and Lmof2365_1120 homologs, annotated as a CAAX aminoterminal putative metalloprotease and AraC-like regulatory protein which share 93.8\% FH2051, 91\% SLCC6466 and SLCC6294 and 91.3\% FH2051, 94\% SLCC6466 and SLCC6294 identity to the L. monocytogenes cluster, respectively. PFGE was carried out to assess the relatedness of the 11 L. innocua strains harbouring intact LIPI-3 a s. On the basis of this analysis, all LIPI- $3^{+}$isolates share a high degree of similarity, with the majority of strains (SLCC6466, SLCC6814, SLCC6749, SLCC6276, SLCC6279, SLCC6294, FH2051, SLCC6296 and SLCC6298) displaying 80\% similarity and strains SLCC6203 and SLCC7199 sharing 76\% identity (Figure 2).

The LIPI- $3^{+}$L. innocua FH2051 is non-haemolytic when grown on Columbia blood agar (Figure 1). This is not surprising given that $L$. innocua strains do not produce LLO and the fact that it has previously been established that LLS is not produced by wild type L. monocytogenes in laboratory media. It has been established that the latter is due to the fact that $\mathrm{P}_{l l s A}$ is not transcribed under standard laboratory conditions [8]. It has been noted previously that $\mathrm{P}_{l l s A}$ is induced under oxidative stress but, unfortunately, the requirement for an oxidizing agent prevents an assessment of associated haemolytic activity on blood agar [7]. Thus, to investigate the functionality of the LIPI-3 cluster in L. innocua, here we constitutively expressed LIPI-3 through the introduction of the constitutive Highly Expressed Listeria Promoter $\left[\mathrm{P}_{\mathrm{HELP}}\left(\mathrm{LLS}^{\mathrm{C}}\right)\right]$ upstream of $l l s A$ in L. innocua FH2051, to create FH2051LLS ${ }^{C}$. Examination of the resultant strain revealed that the L. innocua LIPI-3 is indeed functional as evidenced by a clear haemolytic phenotype on Columbia blood agar (Figure 3).

\section{Conclusion}

In conclusion, we have established that although the presence of the LIPI-3 gene cluster is confined to lineage I isolates of $L$. monocytogenes, a corresponding gene cluster or its remnants can be identified in many $L$. innocua. It is now generally accepted that $L$. innocua and $L$. monocytogenes evolved from a common ancestor, with $L$. innocua having lost virulence genes since this division. Although rare, L. innocua isolates exist which possess the LIPI-1 gene cluster and another $L$. monocytogenes associated virulence gene, inlA [12,13]. Nonetheless, the retention of the LIPI-3 cluster by a large proportion of strains is unexpected. The LIPI-3 clusters in the various $L$. innocua strains seem to be at various stages of reductive evolution with a number of stains possessing an intact island, others showing clear evidence of disintegration and yet another group in which the island is completely absent. It is not clear, however, whether the gradual loss of LIPI-3 from L. innocua strains is a slow process that has been underway since the existence of the last common ancestor of

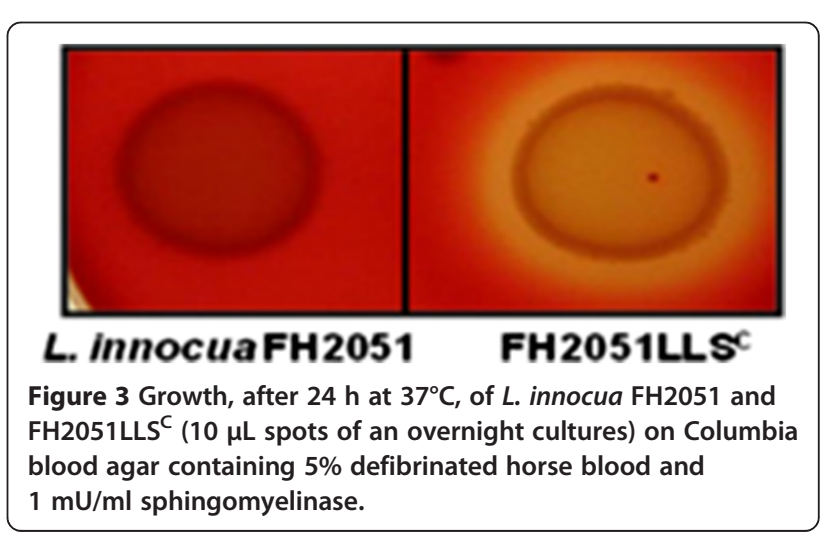


L. monocytogenes and L. innocua or if it was initiated following a more recent acquisition of LIPI-3 by L. innocua from L. monocytogenes.

\section{Competing interests}

The authors have declared that no competing interests exist.

\section{Authors' contributions}

EC contributed to study design, laboratory investigations, data analysis and manuscript preparation, KD contributed to laboratory investigations, data analysis and manuscript preparation, CG contributed to data analysis, PDC, $\mathrm{CH}$ and RPR conceived the study, contributed to study design, data analysis and manuscript preparation. All authors have read and approved the final manuscript.

\section{Acknowledgements}

The authors would like to thank Jana Haase and Mark Achtman for providing strains and Avelino Alvarez Ordonez and Dara Leong for technical assistance with PFGE. This work was funded by the Enterprise Ireland

Commercialisation fund, a programme which is co-financed by the EU through the ERDF. This work was also supported by the Irish Government under the National Development Plan, through Science Foundation Ireland Investigator awards; (06/IN.1/B98) and (10/IN.1/B3027).

\section{Author details}

'Department of Microbiology, University College Cork, Cork, Ireland. ${ }^{2}$ Alimentary Pharmabiotic Centre, Cork, Ireland. ${ }^{3}$ Teagasc, Moorepark Food Research Centre, Fermoy, Co, Cork, Ireland.

Received: 1 August 2013 Accepted: 28 February 2014

Published: 8 March 2014

\section{References}

1. Berche P: Pathophysiology and epidemiology of listeriosis. Bull Acad Natl Med 2005, 189:507-516. discussion 516-21.

2. Hamon M, Bierne H, Cossart P: Listeria monocytogenes: a multifaceted model. Nat Rev Microbiol 2006, 4:423-434.

3. Jackson KA, Iwamoto M, Swerdlow D: Pregnancy-associated listeriosis. Epidemiol Infect 2010, 138:1503-1509.

4. Roberts AJ, Wiedmann M: Pathogen, host and environmental factors contributing to the pathogenesis of listeriosis. Cell Mol Life Sci 2003, 60:904-918.

5. Vazquez-Boland JA, Kuhn M, Berche P, Chakraborty T, Dominguez-Bernal G, Goebel W, Gonzalez-Zorn B, Wehland J, Kreft J: Listeria pathogenesis and molecular virulence determinants. Clin Microbiol Rev 2001, 14:584-640.

6. Orsi RH, den Bakker HC, Wiedmann M: Listeria monocytogenes lineages: genomics, evolution, ecology, and phenotypic characteristics. Int J Med Microbiol 2011, 301:79-96.

7. Clayton EM, Hill C, Cotter PD, Ross RP: Real-time PCR assay to differentiate listeriolysin S-positive and -negative strains of Listeria monocytogenes. Appl Environ Microbiol 2011, 77:163-171.

8. Cotter PD, Draper LA, Lawton EM, Daly KM, Groeger DS, Casey PG, Ross RP, Hill C: Listeriolysin S, a novel peptide haemolysin associated with a subset of lineage I Listeria monocytogenes. PLoS Pathog 2008, 4:e1000144.

9. Molloy EM, Cotter PD, Hill C, Mitchell DA, Ross RP: Streptolysin S-like virulence factors: the continuing sagA. Nature reviews. Microbiology 2011, 9:670-681.

10. den Bakker HC, Bundrant BN, Fortes ED, Orsi RH, Wiedmann M: A population genetics-based and phylogenetic approach to understanding the evolution of virulence in the genus Listeria. Appl Environ Microbiol 2010, 76:6085-6100.

11. den Bakker HC, Cummings CA, Ferreira V, Vatta P, Orsi RH, Degoricija L, Barker M, Petrauskene O, Furtado MR, Wiedmann M: Comparative genomics of the bacterial genus Listeria: genome evolution is characterized by limited gene acquisition and limited gene loss. BMC Genomics 2010, 11:688.

12. Johnson J, Jinneman K, Stelma G, Smith BG, Lye D, Messer J, Ulaszek J, Evsen L, Gendel S, Bennett RW, Swaminathan B, Pruckler J, Steigerwalt A, Kathariou S, Yildirim S, Volokhov D, Rasooly A, Chizhikov V, Wiedmann M, Fortes E, Duvall RE, Hitchins AD: Natural atypical Listeria innocua strains with Listeria monocytogenes pathogenicity island 1 genes. Appl Environ Microbiol 2004, 70:4256-4266.
13. Volokhov DV, Duperrier S, Neverov AA, George J, Buchrieser C, Hitchins AD: The presence of the internalin gene in natural atypically haemolytic Listeria innocua strains suggests descent from L. monocytogenes. Appl Environ Microbiol 2007, 73:1928-1939.

14. Simpson PJ, Stanton C, Fitzgerald GF, Ross RP: Genomic diversity and relatedness of bifidobacteria isolated from a porcine cecum. J Bacteriol 2003, 185:2571-2581

15. Ward TJ, Gorski L, Borucki MK, Mandrell RE, Hutchins J, Pupedis K: Intraspecific phylogeny and lineage group identification based on the prfA virulence gene cluster of Listeria monocytogenes. J Bacteriol 2004, 186:4994-5002.

16. Riedel CU, Monk IR, Casey PG, Morrissey D, O'Sullivan GC, Tangney M, Hill C, Gahan CG: Improved luciferase tagging system for Listeria monocytogenes allows real-time monitoring in vivo and in vitro. Appl Environ Microbiol 2007, 73:3091-3094.

17. Horton RM, Cai ZL, Ho SN, Pease LR: Gene splicing by overlap extension: tailor-made genes using the polymerase chain reaction. Biotechniques 1990, 8:528-535.

18. Monk IR, Gahan CG, Hill C: Tools for functional postgenomic analysis of listeria monocytogenes. Appl Environ Microbiol 2008, 74:3921-3934.

19. Graves ML, Swaminathan B: PulseNet standardized protocol for subtyping Listeria monocytogenes by macrorestriction and pulsed-field gel electrophoresis. Int J Food Microbiol 2001, 65:55-62.

20. Haase JK, Murphy RA, Choudhury KR, Achtman M: Revival of Seeliger's historical 'special Listeria culture Collection'. Environ Microbiol 2011 13:3163.

doi:10.1186/1471-2180-14-58

Cite this article as: Clayton et al:: Atypical Listeria innocua strains possess an intact LIPI-3. BMC Microbiology 2014 14:58.

\section{Submit your next manuscript to BioMed Central and take full advantage of:}

- Convenient online submission

- Thorough peer review

- No space constraints or color figure charges

- Immediate publication on acceptance

- Inclusion in PubMed, CAS, Scopus and Google Scholar

- Research which is freely available for redistribution 